\title{
Long non-coding RNAs act as regulators of cell autophagy in diseases (Review)
}

\author{
ZHIJIE XU ${ }^{1,2^{*}}$, YUANLIANG YAN ${ }^{3, *}$, LONG QIAN $^{3,4}$ and ZHICHENG GONG ${ }^{3,4}$ \\ ${ }^{1}$ Department of Pathology, Xiangya Hospital, ${ }^{2}$ Department of Pathology, School of Basic Medicine, ${ }^{3}$ Department of Pharmacy, \\ Xiangya Hospital, ${ }^{4}$ Institute of Hospital Pharmacy, Central South University, Changsha, Hunan 410008, P.R. China
}

Received September 1, 2016; Accepted January 27, 2017

DOI: $10.3892 /$ or.2017.5416

\begin{abstract}
Identification of long non-coding RNAs (lncRNAs) has provided a substantial increase in our understanding of the non-coding transcriptome. Studies have revealed a crucial function of lncRNAs in the modulation of cell autophagy in vitro and in vivo, further contributing to the hallmarks of disease phenotypes. These findings have profoundly altered our understanding of disease pathobiology, and may lead to the emergence of new biological concepts underlying autophagyassociated diseases, such as the carcinomas. Studies on the molecular mechanism of the lncRNA-autophagy axis may offer additional avenues for therapeutic intervention and biomarker assessment. In this review, we discuss recent findings on the multiple molecular roles of regulatory lncRNAs in the signaling pathways of cell autophagy. The emerging knowledge in this rapidly advancing field will offer novel insights into human diseases, especially cancers.
\end{abstract}

\section{Contents}

1. Introduction

2. The IncRNA-miRNA axis and autophagy

3. Other IncRNAs on autophagy regulation

4. Conclusions and future directions

\section{Introduction}

Autophagy is an evolutionarily conserved process in which cytoplasmic contents are degraded through the cell's own

Correspondence to: Professor Zhicheng Gong, Department of Pharmacy, Xiangya Hospital, Central South University, 87 Xiangya Road, Changsha, Hunan 410008, P.R. China

E-mail: gongzhicheng2013@163.com

${ }^{*}$ Contributed equally

Key words: lncRNAs, autophagy, autophagy-associated diseases, molecular mechanism, cancers lysosomal machinery. Depending on how autophagic substrates are delivered to the lysosome, three different types of autophagy are identified: macroautophagy, microautophagy, and chaperone-mediated autophagy (CMA). As the principal pathway, macroautophagy is used primarily to sequester intact organelles and portions of the cytosol into the autophagosome, a double-membrane vesicle. After the completed autophagosome matures via fusing with the lysosome, its contents are degraded by lysosomal hydrolases. Microautophagy occurs through the direct engulfment of material into the lysosome. CMA, a complex and specific pathway, involves the direct recognition of substrates with the assistance of a lysosomal chaperone (1). Although the above-mentioned classic autophagy pathways are generally considered to be non-selective, accumulating evidence has indicated that specific cargoes can also be degraded selectively (Fig. 1). The discovery and characterization of receptor proteins, such as p62/sequestosome 1 (SQSTM1), have provided pivotal mechanistic insights into this selective autophagic process $(2,3)$.

Since being coined by de Duve in 1963, autophagy has been characterized as an adaptive catabolic process that plays a normal part in cell growth and development, helping to maintain cellular bioenergetic homeostasis (4). In addition, recent studies have revealed other roles for the autophagic machinery in regulating a wide variety of pathological conditions, including tumorigenesis and cardiovascular diseases, suggesting autophagy modulation may have therapeutic value (5-7). However, in the development of human diseases, autophagy has been shown to be a double-edged sword. In some cases, autophagy is a cytoprotective mechanism, but in others, autophagy is a pro-death response to stresses, especially chemotherapy at the cellular and organic levels $(8,9)$. Many autophagy-related genes (Atgs) have been implicated in controlling these complicated behaviors during autophagy, and approximately half of them are evolutionarily conserved from yeast to human (10). Clearly, the regulation of autophagy and the resulting downstream effects are complex and very likely to be cell- and disease type-specific.

Recently, non-coding RNAs, such as long non-coding RNAs (lncRNAs), were demonstrated to regulate cell autophagy in vitro and in vivo, further contributing to many of the hallmarks of disease phenotypes (11) (Fig. 2). The lncRNA highly upregulated liver cancer (HULC) was shown to significantly inhibit cell apoptosis by activating autophagy, 


\section{A NON-SELECTIVE AUtOPHAGY}
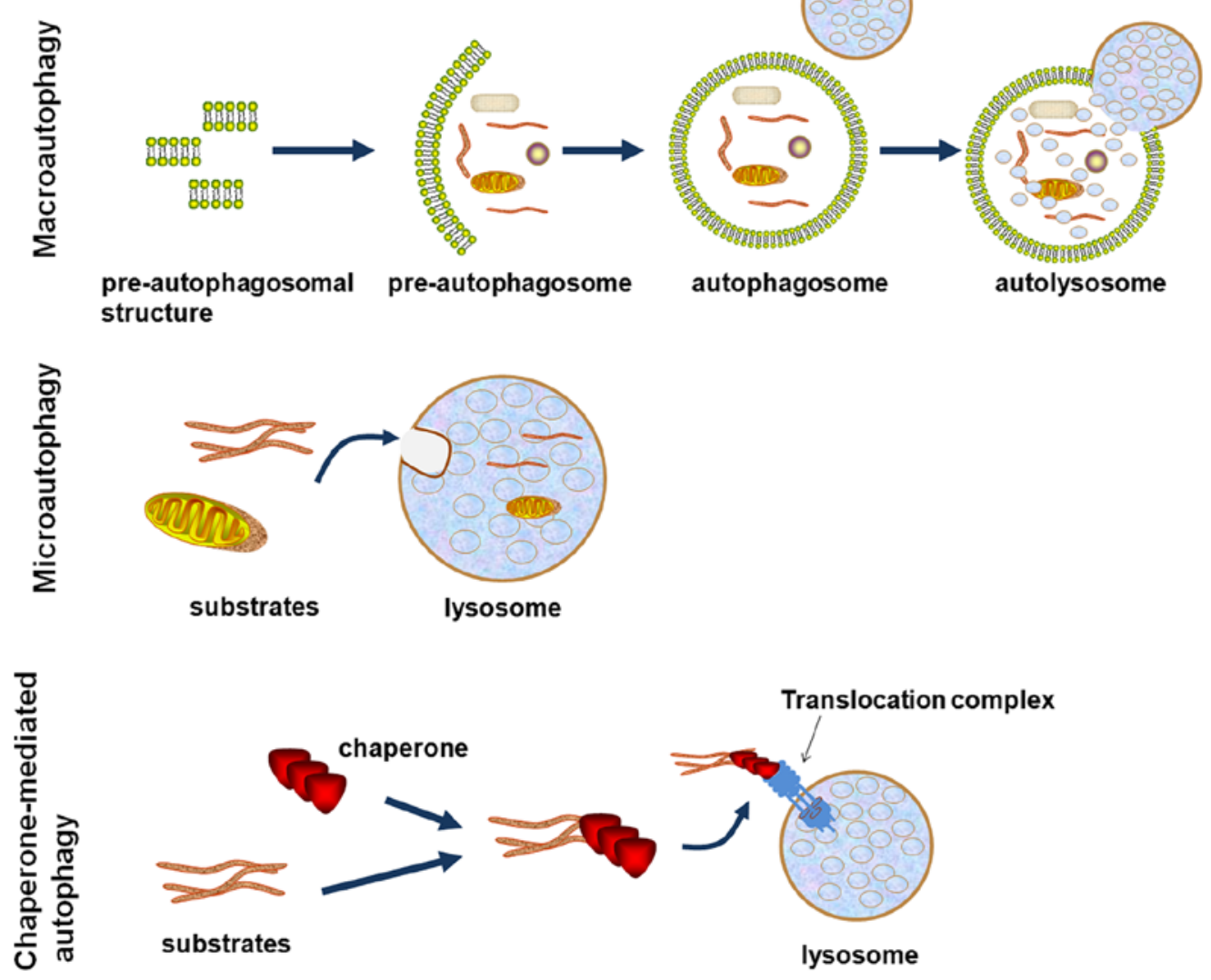

\section{B SELECTIVE AUTOPHAGY}

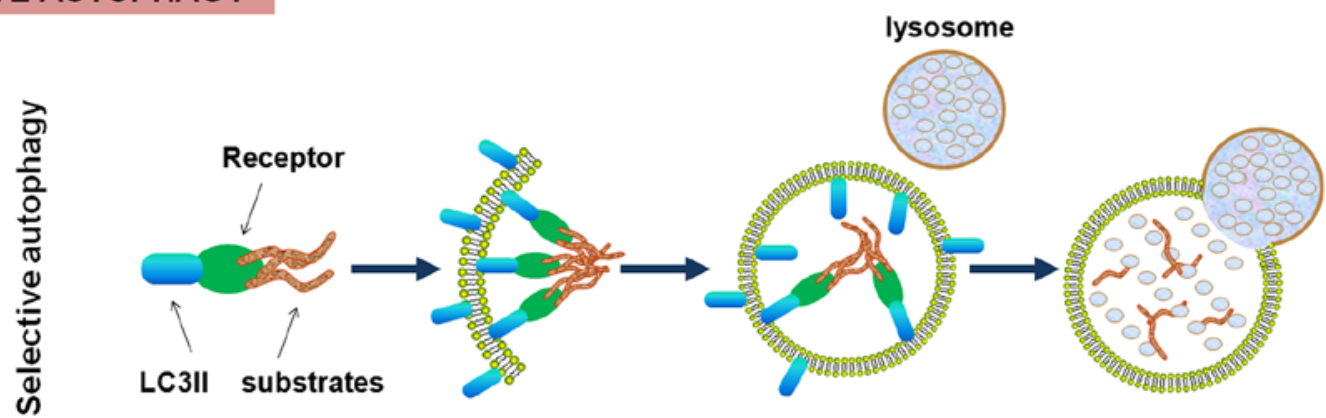

Figure 1. Different modes of cell autophagy. (A) The classic autophagy pathways, such as macroautophagy, microautophagy, and chaperone-mediated autophagy (CMA), are generally considered to be non-selective. (B) Selective autophagy can degrade specific cargoes in a selective manner.

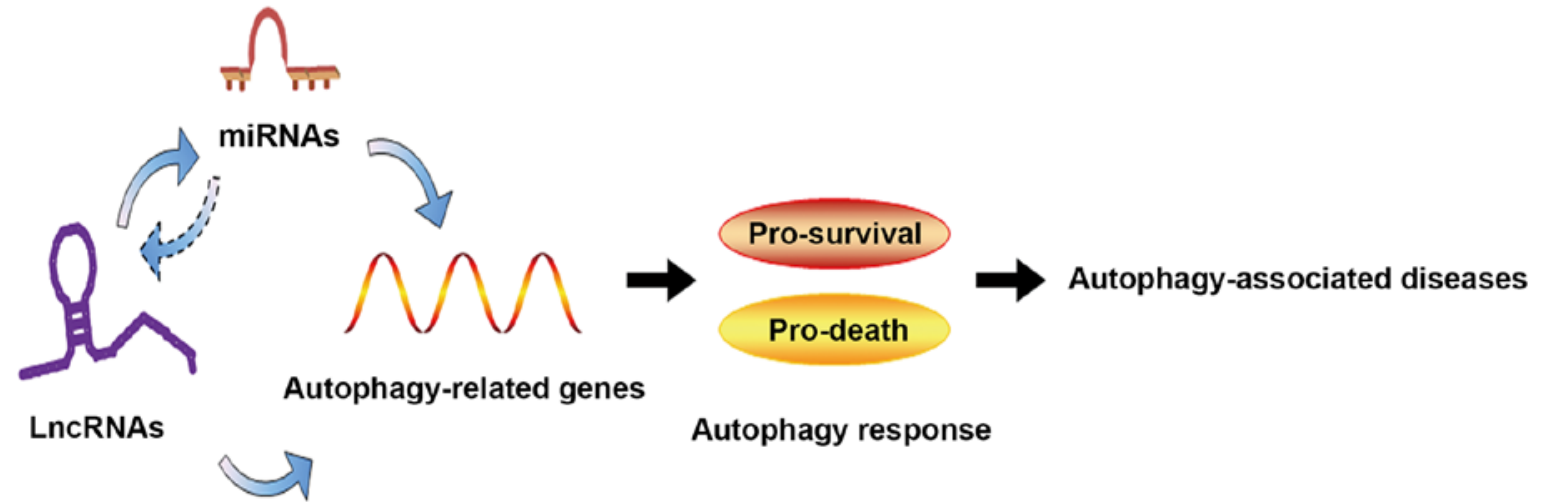

Figure 2. The representative mechanism of lncRNAs on cellular autophagy regulation. The detailed mechanisms are explained in the relevant sections of the text. 
contributing to the malignant phenotype of gastric cancer (12). The lncRNA maternally expressed gene 3 (MEG3), a novel tumor suppressor, was shown to be downregulated in bladder cancer and negatively correlated with microtubule-associated protein 1A/1B-light chain 3 (Atg8/LC3), an autophagy marker. Inhibition of MEG3 by small-interfering RNA (siRNA) activated autophagy, increasing cell proliferation and suppressing cell apoptosis in human bladder cancer cell lines (13). However, in bacterial pathogen infection, the MEG3-autophagy axis has as an opposite effect. In Mycobacterium bovis BCG-infected human macrophages, MEG3 is clearly decreased. Specifically, in silico assays link MEG3 to protein kinase B (Akt) and mammalian target of rapamycin (mTOR) signaling pathways, which can both downregulate cell autophagy. Furthermore, knockdown of MEG3 resulted in the induction of autophagy, which helped to combat the intracellular BCG infection. Upon treatment with IFN- $\gamma$, increased autophagy led to sustained and significant MEG3 downregulation, reinforcing the effective eradication of mycobacteria (14). These investigations have identified a significant relationship between lncRNA and cell autophagy. This review mainly provides an overview of lncRNA biology in autophagy modulation and discusses specific studies that have provided new insight into the underlying mechanism of the lncRNA-autophagy axis for therapeutic intervention.

\section{The IncRNA-miRNA axis and autophagy}

LncRNA-miRNA axis and autophagy. Accumulating studies have highlighted the importance of the non-coding genome in autophagy. Based on length, non-coding RNA can be categorized into several types, such as microRNAs (miRNAs, approximately 22 nucleotides) and lncRNAs ( $>200$ nucleotides). miRNAs have emerged as critical mediators of gene expression through binding to targeted mRNA, including Atgs $(15,16)$. As the dysregulated expression of Atgs has been shown to have a far reaching impact on human diseases, miRNAs are being examined as potential novel biomarkers or therapeutic targets (17). One particular example is the miR-30a family, which is linked to autophagy and various pathophysiological conditions. miR-30a negatively regulates BECN1/Atg6 stability via the predicted binding sequence in its $3^{\prime}$ untranslated region (UTR). Downregulation of miRNA-30a increased the mRNA and protein levels of BECN1, markedly initiating basal autophagy in mature adipocytes (18). Similarly, miR-30a inhibition alleviated ischemia/reperfusion-induced neurological dysfunction through enhancing BECN1-mediated autophagy (19). Additionally, miRNA-30a-5p can target metadherin (MTDH) mRNA for cleavage or translational repression. By targeting MTDH, miRNA-30a-5p downregulated activation of the Akt signaling pathway, which might regulate autophagy and apoptosis of cancer cells $(20,21)$. In another study, identical recognition sites of miR-137 were found at the 3'UTRs of the selective autophagy receptors NIX/BNIP3L (BCL2/ adenovirus E1B $19 \mathrm{kDa}$ interacting protein 3-like) and FUN14 domain containing 1 (FUNDC1). In response to hypoxia, miR-137 reduced the expression of Nix and FUNDC1, thereby inhibiting mitophagy, a selective autophagy of impaired mitochondria, without affecting classic autophagy (22). However, the exact role of different miRNAs in regulating classic or selective autophagy must still be elucidated. Gibbings et al demonstrated that selective autophagy can in turn modulate miRNA homeostasis. The miRNA-processing enzyme Dicer and the miRNA effector Argonaute 2 (Ago2) are critical components responsible for miRNA synthesis. Under the control of the autophagy receptor nuclear dot protein $52 \mathrm{kDa}$ (NDP52), Dicer and Ago2 were targeted for degradation by selective autophagy, which inhibited homeostasis and activity of the targeted miRNAs $(23,24)$. These data have profound implications for the association of miRNA-levels with dysregulated autophagy.

The role of miRNAs in the regulation of autophagy by $\operatorname{lncRNA}$. Bioinformatics analysis of molecular interactions has identified miRNA recognition elements (MREs) on lncRNA sequences, demonstrating the direct transcriptional regulation of miRNA by lncRNA (25). Furthermore, perturbation of lncRNA expression disrupted the balance of IncRNA-miRNA regulatory paradigms, contributing to the pathogenesis of various diseases, including cancers. To date, four forms of functional cross-regulation have been artificially identified $(26,27)$ (Fig. 3). The stability of IncRNAs can be weakened through interaction with specific miRNAs. Yoon et al found that the stability of IncRNA p21 was controlled by the miRNA let-7b. Overexpression of precursor-let-7 decreased the half-life of lncRNA p21 and accelerated its degradation (28). Likewise, another IncRNA, HOX antisense intergenic RNA (HOTAIR), was shown to be enriched and stable after using an antagomir to inhibit endogenous let-7i in human cervical carcinoma HeLa cells (29). LncRNAs can function as miRNA decoys or sponges, also known as competitive endogenous RNAs (ceRNAs). The function of lncRNAs as ceRNAs significantly expands their regulatory capacity; ceRNAs can compete with mRNA for the binding of miRNA and thus repress the target gene at the post-transcriptional level. Furthermore, numerous lines of bioinformatics evidence from several well-known groups have supported the ceRNA function of IncRNAs, including the database of human long non-coding RNA acting as competing endogenous RNA (lnCeDB) (30), the lncRNA-associated competing triplet database (LncACTdb) (31) and the database of cancer somatic mutations altering microRNA-ceRNA interactions (SomamiR 2.0) (32). LncRNAs can compete with miRNAs for interaction with shared target mRNAs. Franklin et al revealed that the long non-coding N-ras functional RNA (ncNRFR) competes with let-7 and reverses the repression of let-7 site-bearing mRNAs (33). Faghihi et al found a miR-485-5p binding site in the mRNA of $\beta$-site amyloid precursor protein cleaving enzyme 1 (BACE1). By blocking access of miR-485-5p, the lncRNA BACE1-antisense transcript prevented miRNA-induced suppression of BACE1 mRNA (34). LncRNAs are a source of miRNAs for inducing target mRNA silencing. Exon 1 of lncRNA H19 encodes two conserved miRNAs, miR-675-3p and miR-675-5p. Exogenous miR-675-3p and miR-675-5p both rescue abnormal skeletal muscle regeneration induced by $\mathrm{H} 19$ depletion (35). In summary, the lncRNA-miRNA axis controls gene expression patterns driving many cellular processes, such as cell development, proliferation, and cell autophagy.

It is well known that mTOR, a central negative-regulator of cell autophagy, plays a key role in regulating the balance 
A miRNAs-weakened IncRNAs stability

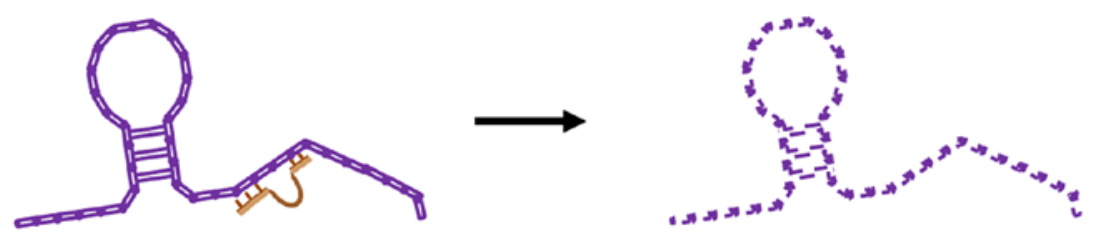

B LncRNAs functions as miRNA decoys or sponges

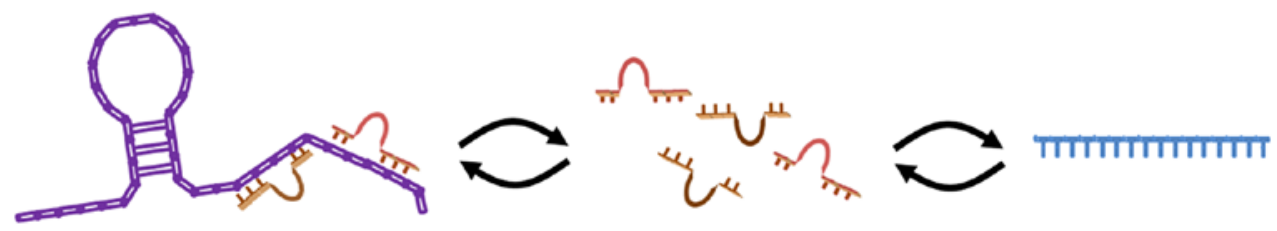

\section{LncRNAs compete with miRNAs for targeted mRNAs}
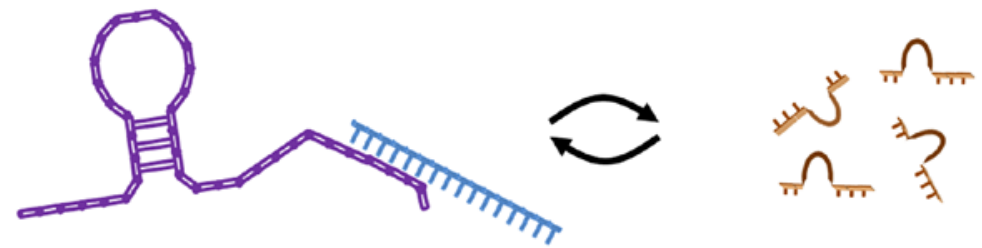

D LncRNAs as a source of miRNAs
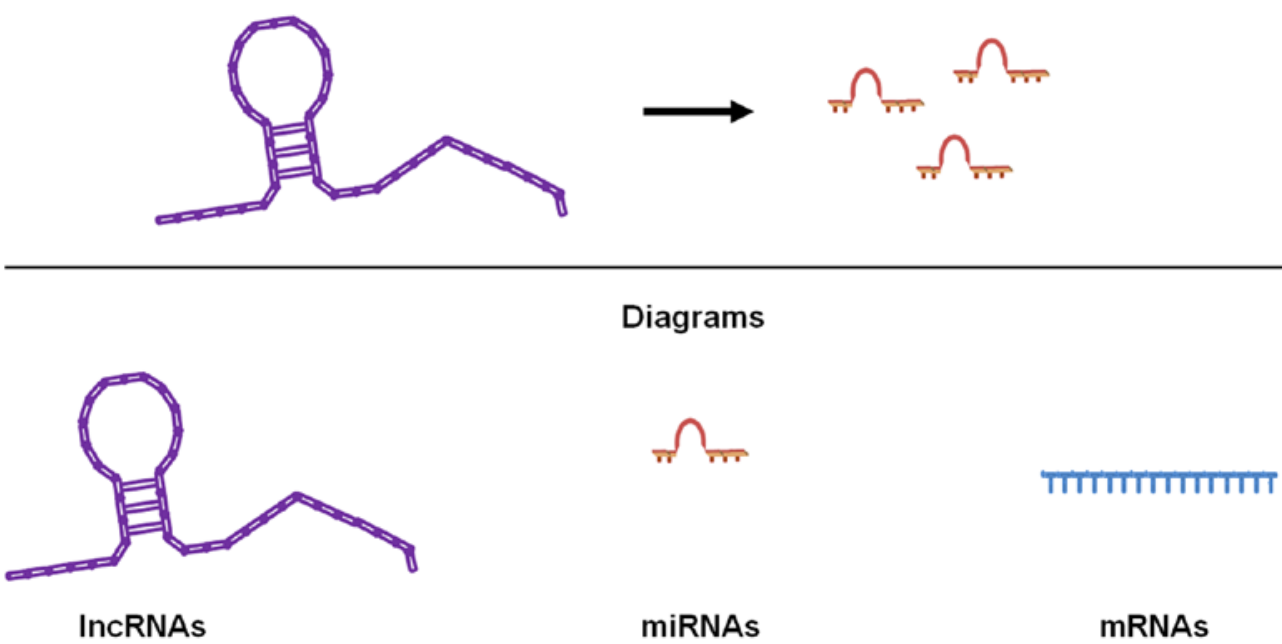

Figure 3. Schematic of the major forms of interactions between lncRNAs and miRNAs.

between cell growth and death in response to stress signals. Modulating abnormal mTOR signaling is important for numerous physiological and pathological conditions (36). After treatment with doxorubicin, mTOR activation via suppression of ELK3 impaired autophagy, and thus enhanced cell viability in the breast cancer cell line MDA-MB-231 (37). Using molecular docking prediction, Ge et al identified a novel mTOR activator, 3-benzyl-5-((2-nitrophenoxy) methyl)-dihydrofuran-2(3H)-one (3BDO). They found that activation of mTOR induced by 3BDO increased phosphorylation of T-cell-restricted intracellular antigen-1 (TIA1), which is responsible for processing FLJ11812, a nuclear lncRNA derived from the 3'UTR of transforming growth factor $\beta 2$ (TGFß2). Acting as a ceRNA, FLJ11812 competed for binding with miR-4459, resulting in the increase of its target Atg13 and promotion of autophagy (38). Apart from miR-4459, FLJ11812 also acts as the molecular decoy of miR-3960 and miR-4488, elevating the expression of miRNA targets ceramide synthase 1 (CERS1) and N-acetyltransferase 8-like (NAT8L), respectively, two molecules participating in mitophagy (39). In addition, phospholipase D (PLD) inhibitor stimulates cell autophagy and exhibits attractive antitumorigenic effects by destabilizing mTOR. The IncRNA antisense non-coding RNA in the INK4 locus (ANRIL) has been shown to be responsible for the functions of PLD inhibitor (40). In sleeping beauty (SB)-baculovirus (BV) systems, ectopic expression of the lncRNA phosphatase and tensin (PTEN) homolog pseudogene 1 (PTENP1) in hepatocellular carcinoma suppressed 
the Akt/mTOR signaling pathway by promoting PTEN transcription, thus inducing autophagic associated cell death. Moreover, PTENP1 also acted as a decoy for miR-17, miR-19b, and miR-20a, which enhanced autophagy of their target genes Atg7 and Unc-51 like autophagy activating kinase 1 (ULK1) (41). While most current studies mainly focus on the function of lncRNAs in the nucleus, Liu et al recently uncovered a function of the cytoplasmic lncRNA neighbor of BRCA1 gene 2 (NBR2). Energy stress could promote the colocalization of NBR2 and AMPK in the cytoplasm, potentiating AMPK activation. Finally, knockdown of NBR2 attenuated stress-induced mTOR inactivation by repressing the AMPK kinase signal, thus altering the autophagy response and increasing tumor development (42). Together, these results indicate that mTOR activation can inhibit cell autophagy via lncRNA-miRNA signaling, leading to participation in autophagy-mediated processes, such asembryogenesis (43), atherosclerosis (44), and tumorigenesis (41).

Apart from mTOR, the IncRNA-miRNA axis also directly affects Atgs modulation. As a direct target of miR-21, the lncRNA growth arrest-specific 5 (GAS5) is upregulated during osteoarthritis. Overexpression of GAS5 inhibited cell autophagy, as indicated by downregulation of the LC3-II/LC3-I ratio and $\mathrm{BECN} 1$, and stimulated the death of articular chondrocytes (45). Recently, Liu et al defined another growthpromoted lncRNA in hepatocellular carcinoma, HNF1A-AS1 (antisense transcript of HNF1A). Oncogenic HNF1A-AS1 sequestered miR-30b in a ceRNA-dependent manner and stimulated autophagy flux via promoting the formation of the Atg12 complex, thus contributing to hepatocarcinogenesis (46). In ischemia/reperfusion-induced myocardial infarction, Atg7, a specific target of miR-188-3p, is upregulated, leading to autophagic cell death. Through lncRNA array analysis, Wang et al reported a novel lncRNA, autophagy promoting factor (APF). APF regulated the expression of Atg7 and the consequent autophagy by sponging miR-188-3p (47). Together, these data indicate critical roles of IncRNAs in cell autophagy as well as autophagic-associated diseases.

\section{Other IncRNAs on autophagy regulation}

The human PVT1 gene is a lncRNA on chromosome 8q24 and is critical in the regulation of cell growth, and differentiation. Many studies have demonstrated that the biological function of PVT1 mainly depends on two mechanisms. First, PVT1 inhibits gene promoter activity. Zhang et al found PVT1 was an oncogenic gene in cervical cancer. Ectopic expression of PVT1 recruited enhancer of zeste homolog 2 (EZH2), a member of the polycomb-group (PcG) family, to the miR-200b promoter and repressed miR-200b expression by methylating Lys-27 (H3K27me) of histone H3 on the miR-200b promoter (48). Similarly, PVT1 repressed large tumor suppressor kinase 2 (LATS2) transcription by recruiting EZH2 to the LASTS2 promoter, facilitating cell proliferation in non-small cell lung cancer (49). Secondly, PVT1 regulates protein stability. As the PVT1 locus is adjacent to the c-Myc locus, PVT1 is required for high Myc protein levels in cancer cells. According to a study by Tseng et al, PVT1 controlled Myc levels through regulation of protein stability (50). Furthermore, knockdown of PVT1 by RNA interference led to inhibition of cancer cell proliferation by suppressing the Myc protein level (51). Additionally, another group studied PVT1-mediated autophagy in cognitive impairment of diabetes mellitus. They found that autophagy inhibitor 3-methyladenine (3-MA) could significantly induce apoptotic cell death by decreasing hippocampal PVT1 expression, which subsequently heightened cognitive impairment (52). This was the first time that lncRNA homeostasis was shown to be controlled by cell autophagy, but the specific mechanisms need to be further elucidated.

BRAF-activated lncRNA (BANCR), a 693-bp transcripton chromosome 9 , is a useful biomarker or future therapeutic target in various cancers, including melanoma (53), papillary thyroid carcinoma (54), and lung cancer (55). Flockhart et al and Wajapeyee et al performed massively parallel cDNA sequencing (RNA-seq) and found that the expression of BANCR was regulated by mutant BRAFV600E protein, the most prevalent mutation of the BRAF gene in the circulating tumor DNA of a patient $(56,57)$. A recent study demonstrated that the autophagy inhibitors VATG-027 and VATG-032, which are acridine and tetrahydroacridine derivatives of the antimalarial agent chloroquine, significantly enhanced the sensitivity of cancer cells to the BRAF V600E inhibitor vemurafenib (58). Moreover, selective BRAFV600E inhibitors induced autophagy by activating AMPK signaling in colorectal cancer cells (59). As BANCR is shown to be strongly linked with BRAFV600E mutation, BANCR may have a possible role in autophagy modulation. As expected, overexpression of BANCR in papillary thyroid carcinoma enhanced the ratio of LC3-II/LC3-I, a marker for autophagy, resulting in increased cell proliferation and inhibited apoptosis (60).

Concerning cardiac remodeling, Viereck and colleagues performed global lncRNA expression profiling and identified the IncRNA cardiac hypertrophy-associated transcript (Chast) as a potential influence on cardiomyocyte hypertrophy in vivo. GapmeR-mediated Chast silencing upregulates Atg5 and increases the LC3-II/LC3-I ratio, driving cell autophagy capacity and impeding the pathologic remodeling processes. Bioinformatics analysis of gene sequence revealed partial overlaps between Chast and the antisense strand of pleckstrin homology domain-containing protein family $\mathrm{M}$ member 1 (plekhm1), a regulator of autophagy, suggesting that Chast might downregulate cell autophagy by a plekhm1-dependent mechanism (61).

\section{Conclusions and future directions}

Advances in sequencing technologies have identified numerous lncRNAs. Though previously considered 'junk sequences' in our genomes, the epigenetic role of IncRNAs promises to be another exciting frontier for disease research and therapy. The discovery of lncRNAs and their functions has shed light on novel molecular mechanisms of autophagy regulation, potentially improving the diagnosis and treatment of human autophagy-associated diseases (Table I). Although the precise cellular mechanism of IncRNA is not completely understood, recent studies have revealed the existence of stable lncRNAs protected from endogenous RNases in blood and other bodily fluids of patients, the so-called circulating lncRNAs (62). Due to growing interest in the function of lncRNAs as diagnostic and prognostic biomarkers, lncRNAs are increasingly gaining 
Table I. Examples of lncRNAs associated with autophagy regulations.

\begin{tabular}{llllr}
\hline IncRNA & Locus & \multicolumn{1}{c}{ Role in autophagy } & \multicolumn{1}{c}{ Related diseases } & Refs. \\
\hline HULC & Chr6 & Increasing LC3-II/LC3-I ratio & Gastric cancer & $(12)$ \\
MEG3 & Chr14 & $\begin{array}{l}\text { Negatively correlateing with LC3 } \\
\text { Promoting Akt/mTOR signal pathways }\end{array}$ & Bladder cancer & $(13)$ \\
& & Bacterial infection & $(14)$ \\
FLJ11812 & Chr1 & Increasing Atg13 level & VECs dysfunction & $(40)$ \\
ANRIL & Chr9 & Increasing LC3-II/LC3-I ratio & Lung cancer & $(41)$ \\
PTENP1 & Chr9 & Suppressing Akt/mTOR signal pathways & Hepatocellular & $(42)$ \\
& & Increasing Atg7 and ULK1 level & carcinoma & $(43)$ \\
NBR2 & Chr17 & Activating the cytoplasmic AMPK & Several cancers & $(46)$ \\
GAS5 & Chr1 & Suppressing LC3-II/LC3-I ratio and BECN1 & Osteoarthritis & $(47)$ \\
HNF1A-AS1 & Chr12 & promoting the formation of Atg12 complex & Hepatocellular carcinoma \\
APF & Chr8 & Increasing Atg7 level & Myocardial infarction & $(48)$ \\
PVT1 & Chr8 & Downregulated by autophagy inhibitor & Diabetes mellitus & $(53)$ \\
BANCR & Chr9 & Increasing LC3-II/LC3-I ratio & Papillary thyroid carcinoma & $(61)$ \\
Chast & Chr11 & Suppressing LC3-II/LC3-I ratio, BECN1 and plekhm1 & Cardiomyocyte hypertrophy & $(62)$ \\
\hline Chr, chromosome; & LC3, microtubule-associated protein 1A/1B-light chain 3; Akt, protein kinase B; mTOR, mammalian target of rapamycin; \\
VECs, vascular endothelial cells; ULK1, Unc-51 like autophagy activating kinase 1; plekhm1, pleckstrin homology domain-containing protein \\
family M member 1.
\end{tabular}

the attention of scientists and clinicians. However, two critical issues must still be solved: uniformity of sample preparation and data normalization (63). In addition, lncRNAs in extracellular vesicles, such as exosomes, could offer a novel, enriched source of IncRNAs. These exosomes have been found to harbor disease-derived specific lncRNAs, which were significantly different between patients and normal controls (64). Qu et al found that the exosome-transmitted lncRNA activated in renal cell carcinoma with sunitinib resistance (ARSR) potentially affects the chemosensitivity of cancer cells (65). In addition, the regulation of cell autophagy by circulating lncRNAs under pathophysiological conditions needs to be discussed. The exciting potential of these emerging lncRNAs as biomarkers could be an important advance in disease management.

Autophagy, a tightly regulated catabolic process of cellular self-digestion, can be activated by several stresses, especially pharmacological action, and constitutes a potential target for disease therapy $(66,67)$. Studies have indicated that autophagy appears to serve as either a pro-survival or pro-death response to therapeutic treatment (68). However, the precise role of autophagy in modulating cell biological behavior is highly dependent on the cellular context and its extent. A recent study found that lncRNAs can also influence drug-sensitivity by modulating cell autophagy. Overexpression of the IncRNA regulator of insulin sensitivity and autophagy (Risa) in hepatocytes or myotubes significantly decreased autophagy, thus attenuating insulin resistance. Knockdown of Atg7 or Atg5 by siRNAs inhibited the effect of Risa on insulin resistance in vivo (69). These findings show a potentially attractive approach to improving the curative effect: modulating the IncRNA-autophagy axis. Thus, further investigation of lncRNAs in autophagy regulation may identify novel strategies to enhance the benefits of pharmacotherapy in the treatment of human diseases.

\section{Acknowledgements}

This study was supported by the National Natural Science Foundation of China (no. 81572946), the Changsha Science and Technology Project (no. k1508024-31), and the Clinical and Rehabilitation Research Foundation of Xiangya Hospital Beidaweiming.

\section{References}

1. Jia G and Sowers JR: Autophagy: A housekeeper in cardiorenal metabolic health and disease. Biochim Biophys Acta 1852: 219-224, 2015

2. Wurzer B, Zaffagnini G, Fracchiolla D, Turco E, Abert C, Romanov J and Martens S: Oligomerization of p62 allows for selection of ubiquitinated cargo and isolation membrane during selective autophagy. eLife 4: e08941, 2015.

3. Ryter SW, Cloonan SM and Choi AM: Autophagy: A critical regulator of cellular metabolism and homeostasis. Mol Cells 36: 7-16, 2013.

4. Galluzzi L, Bravo-San Pedro JM, Blomgren K and Kroemer G: Autophagy in acute brain injury. Nat Rev Neurosci 17: 467-484, 2016.

5. Roos WP, Thomas AD and Kaina B: DNA damage and the balance between survival and death in cancer biology. Nat Rev Cancer 16: 20-33, 2016.

6. Lorenzen JM and Thum T: Long noncoding RNAs in kidney and cardiovascular diseases. Nat Rev Nephrol 12: 360-373, 2016.

7. Bento CF, Renna M, Ghislat G, Puri C, Ashkenazi A, Vicinanza M, Menzies FM and Rubinsztein DC: Mammalian autophagy: How does it work? Annu Rev Biochem 85: 685-713, 2016.

8. Evangelisti C, Evangelisti C, Chiarini F, Lonetti A, Buontempo F, Neri LM, McCubrey JA and Martelli AM: Autophagy in acute leukemias: A double-edged sword with important therapeutic implications. Biochim Biophys Acta 1853: 14-26, 2015. 
9. Chen Y, Henson ES, Xiao W, Huang D, McMillan-Ward EM, Israels SJ and Gibson SB: Tyrosine kinase receptor EGFR regulates the switch in cancer cells between cell survival and cell death induced by autophagy in hypoxia. Autophagy 12 : 1029-1046, 2016

10. Xie Y, Kang R, Sun X, Zhong M, Huang J, Klionsky DJ and Tang D: Posttranslational modification of autophagy-related proteins in macroautophagy. Autophagy 11: 28-45, 2015.

11. Choudhry H, Harris AL and McIntyre A: The tumour hypoxia induced non-coding transcriptome. Mol Aspects Med 47-48: 35-53, 2016.

12. Zhao Y, Guo Q, Chen J, Hu J, Wang S and Sun Y: Role of long non-coding RNA HULC in cell proliferation, apoptosis and tumor metastasis of gastric cancer: A clinical and in vitro investigation. Oncol Rep 31: 358-364, 2014

13. Ying L, Huang Y, Chen H, Wang Y, Xia L, Chen Y, Liu Y and Qiu F: Downregulated MEG3 activates autophagy and increases cell proliferation in bladder cancer. Mol Biosyst 9: 407-411, 2013.

14. Pawar K, Hanisch C, Palma Vera SE, Einspanier R and Sharbati S Down regulated lncRNA MEG3 eliminates mycobacteria in macrophages via autophagy. Sci Rep 6: 19416, 2016.

15. Hall DP, Cost NG, Hegde S, Kellner E, Mikhaylova O, Stratton Y, Ehmer B, Abplanalp WA, Pandey R, Biesiada J, et al: TRPM3 and miR-204 establish a regulatory circuit that controls oncogenic autophagy in clear cell renal cell carcinoma. Cancer Cell 26: 738-753, 2014

16. Piccoli MT, Gupta SK and Thum T: Noncoding RNAs as regulators of cardiomyocyte proliferation and death. J Mol Cell Cardiol 89 (Pt A): 59-67, 2015.

17. Ouimet M, Koster S, Sakowski E, Ramkhelawon B, van Solingen C, Oldebeken S, Karunakaran D, Portal-Celhay C, Sheedy FJ, Ray TD, et al: Mycobacterium tuberculosis induces the miR-33 locus to reprogram autophagy and host lipid metabolism. Nat Immunol 17: 677-686, 2016

18. Deng Y, Xu J, Zhang X, Yang J, Zhang D, Huang J, Lv P, Shen W and Yang Y: Berberine attenuates autophagy in adipocytes by targeting BECN1. Autophagy 10: 1776-1786, 2014.

19. Wang P, Liang J, Li Y, Li J, Yang X, Zhang X, Han S, Li S and Li J: Down-regulation of miRNA-30a alleviates cerebral ischemic injury through enhancing beclin 1-mediated autophagy. Neurochem Res 39: 1279-1291, 2014.

20. Li WF, Dai H, Ou Q, Zuo GQ and Liu CA: Overexpression of microRNA-30a-5p inhibits liver cancer cell proliferation and induces apoptosis by targeting MTDH/PTEN/AKT pathway. Tumour Biol 37: 5885-5895, 2016.

21. Wang RC, Wei Y, An Z, Zou Z, Xiao G, Bhagat G, White M, Reichelt $\mathbf{J}$ and Levine B: Akt-mediated regulation of autophagy and tumorigenesis through Beclin 1 phosphorylation. Science 338: 956-959, 2012

22. Li W, Zhang X, Zhuang H, Chen HG, Chen Y, Tian W, Wu W, Li Y, Wang S, Zhang L, et al: MicroRNA-137 is a novel hypoxiaresponsive microRNA that inhibits mitophagy via regulation of two mitophagy receptors FUNDC1 and NIX. J Biol Chem 289: 10691-10701, 2014.

23. Gibbings D, Mostowy S, Jay F, Schwab Y, Cossart P and Voinnet O: Selective autophagy degrades DICER and AGO2 and regulates miRNA activity. Nat Cell Biol 14: 1314-1321, 2012.

24. Gibbings D, Mostowy S and Voinnet O: Autophagy selectively regulates miRNA homeostasis. Autophagy 9: 781-783, 2013.

25. Paraskevopoulou MD, Vlachos IS, Karagkouni D, Georgakilas G, Kanellos I, Vergoulis T, Zagganas K, Tsanakas P, Floros E, Dalamagas T, et al: DIANA-LncBase v2: Indexing microRNA targets on non-coding transcripts. Nucleic Acids Res 44 (D1) D231-D238, 2016

26. Yoon JH, Abdelmohsen K and Gorospe M: Functional interactions among microRNAs and long noncoding RNAs. Semin Cell Dev Biol 34: 9-14, 2014

27. Ballantyne MD, McDonald RA and Baker AH: lncRNA/ MicroRNA interactions in the vasculature. Clin Pharmacol Ther 99: 494-501, 2016

28. Yoon JH, Abdelmohsen K, Srikantan S, Yang X, Martindale JL, De S, Huarte M, Zhan M, Becker KG and Gorospe M: LincRNAp21 suppresses target mRNA translation. Mol Cell 47: 648-655, 2012.

29. Yoon JH, Abdelmohsen K, Kim J, Yang X, Martindale JL, Tominaga-Yamanaka K, White EJ, Orjalo AV, Rinn JL, Kreft SG, et al: Scaffold function of long non-coding RNA HOTAIR in protein ubiquitination. Nat Commun 4: 2939, 2013.
30. Das S, Ghosal S, Sen R and Chakrabarti J: lnCeDB: Database of human long noncoding RNA acting as competing endogenous RNA. PLoS One 9: e98965, 2014.

31. Wang P, Ning S, Zhang Y, Li R, Ye J, Zhao Z, Zhi H, Wang T, Guo $Z$ and $L i X$ : Identification of lncRNA-associated competing triplets reveals global patterns and prognostic markers for cancer. Nucleic Acids Res 43: 3478-3489, 2015.

32. Bhattacharya A and Cui Y: SomamiR 2.0: A database of cancer somatic mutations altering microRNA-ceRNA interactions. Nucleic Acids Res 44 (D1): D1005-D1010, 2016.

33. Franklin JL, Rankin CR, Levy S, Snoddy JR, Zhang B, Washington MK, Thomson JM, Whitehead RH and Coffey RJ: Malignant transformation of colonic epithelial cells by a colonderived long noncoding RNA. Biochem Biophys Res Commun 440: 99-104, 2013

34. Faghihi MA, Zhang M, Huang J, Modarresi F, Van der Brug MP, Nalls MA, Cookson MR, St-Laurent G III and Wahlestedt C: Evidence for natural antisense transcript-mediated inhibition of microRNA function. Genome Biol 11: R56, 2010.

35. Dey BK, Pfeifer K and Dutta A: The H19 long noncoding RNA gives rise to microRNAs miR-675-3p and miR-675-5p to promote skeletal muscle differentiation and regeneration. Genes Dev 28: 491-501, 2014.

36. Gallagher LE, Williamson LE and Chan EY: Advances in autophagy regulatory mechanisms. Cells 5: 24, 2016.

37. Park JH, Kim KP, Ko JJ and Park KS: PI3K/Akt/mTOR activation by suppression of ELK3 mediates chemosensitivity of MDA-MB-231 cells to doxorubicin by inhibiting autophagy. Biochem Biophys Res Commun 477: 277-282, 2016.

38. Ge D, Han L, Huang S, Peng N, Wang P, Jiang Z, Zhao J, Su L, Zhang S, Zhang Y, et al: Identification of a novel MTOR activator and discovery of a competing endogenous RNA regulating autophagy in vascular endothelial cells. Autophagy 10: 957-971, 2014.

39. Huang S, Lu W, Ge D, Meng N, Li Y, Su L, Zhang S, Zhang Y, Zhao B and Miao J: A new microRNA signal pathway regulated by long noncoding RNA TGFB2-OT1 in autophagy and inflammation of vascular endothelial cells. Autophagy 11: 2172-2183, 2015.

40. Kang YH, Kim D and Jin EJ: Down-regulation of phospholipase D stimulates death of lung cancer cells involving up-regulation of the long ncRNA ANRIL. Anticancer Res 35: 2795-2803, 2015

41. Chen CL, Tseng YW, Wu JC, Chen GY, Lin KC, Hwang SM and $\mathrm{Hu}$ YC: Suppression of hepatocellular carcinoma by baculovirusmediated expression of long non-coding RNA PTENP1 and microRNA regulation. Biomaterials 44: 71-81, 2015.

42. Liu X, Xiao ZD, Han L, Zhang J, Lee SW, Wang W, Lee H, Zhuang L, Chen J, Lin HK, et al: LncRNA NBR 2 engages a metabolic checkpoint by regulating AMPK under energy stress. Nat Cell Biol 18: 431-442, 2016.

43. Lu W, Han L, Su L, Zhao J, Zhang Y, Zhang S, Zhao B and Miao J: A 3'UTR-associated RNA, FLJ11812 maintains stemness of human embryonic stem cells by targeting miR-4459. Stem Cells Dev 24: 1133-1140, 2015.

44. Peng N, Meng N, Wang S, Zhao F, Zhao J, Su L, Zhang S, Zhang Y, Zhao B and Miao J: An activator of mTOR inhibits oxLDL-induced autophagy and apoptosis in vascular endothelial cells and restricts atherosclerosis in apolipoprotein $\mathrm{E}^{-/ 2}$ mice. Sci Rep 4: 5519, 2014

45. Song J, Ahn C, Chun CH and Jin EJ: A long non-coding RNA, GAS5, plays a critical role in the regulation of miR-21 during osteoarthritis. J Orthop Res 32: 1628-1635, 2014.

46. Liu Z, Wei X, Zhang A, Li C, Bai J and Dong J: Long non-coding RNA HNF1A-AS1 functioned as an oncogene and autophagy promoter in hepatocellular carcinoma through sponging hsamiR-30b-5p. Biochem Biophys Res Commun 473: 1268-1275, 2016.

47. Wang K, Liu CY, Zhou LY, Wang JX, Wang M, Zhao B, Zhao WK, Xu SJ, Fan LH, Zhang XJ, et al: APF lncRNA regulates autophagy and myocardial infarction by targeting miR-188-3p. Nat Commun 6: 6779, 2015.

48. Zhang S, Zhang G and Liu J: Long noncoding RNA PVT1 promotes cervical cancer progression through epigenetically silencing miR-200b. APMIS 124: 649-658, 2016.

49. Wan L, Sun M, Liu GJ, Wei CC, Zhang EB, Kong R, Xu TP, Huang MD and Wang ZX: Long noncoding RNA PVT1 promotes non-small cell lung cancer cell proliferation through epigenetically regulating LATS2 expression. Mol Cancer Ther 15: $1082-1094,2016$ 
50. Tseng YY, Moriarity BS, Gong W, Akiyama R, Tiwari A, Kawakami H, Ronning P, Reuland B, Guenther K, Beadnell TC, et al: PVT1 dependence in cancer with MYC copy-number increase. Nature 512: 82-86, 2014

51. Zeng C, Yu X, Lai J, Yang L, Chen S and Li Y: Overexpression of the long non-coding RNA PVT1 is correlated with leukemic cell proliferation in acute promyelocytic leukemia. J Hematol Oncol 8: 126, 2015.

52. Li Z, Hao S, Yin H, Gao J and Yang Z: Autophagy ameliorates cognitive impairment through activation of PVT1 and apoptosis in diabetes mice. Behav Brain Res 305: 265-277, 2016.

53. McCarthy N: Epigenetics. Going places with BANCR. Nat Rev Cancer 12: 451, 2012.

54. Zheng H, Wang M, Jiang L, Chu H, Hu J, Ning J, Li B, Wang D and $\mathrm{Xu}$ J: BRAF-activated long noncoding RNA modulates papillary thyroid carcinoma cell proliferation through regulating thyroid stimulating hormone receptor. Cancer Res Treat 48: 698-707, 2016

55. Sun M, Liu XH, Wang KM, Nie FQ, Kong R, Yang JS, Xia R, $\mathrm{Xu}$ TP, Jin FY, Liu ZJ, et al: Downregulation of BRAF activated non-coding RNA is associated with poor prognosis for non-small cell lung cancer and promotes metastasis by affecting epithelialmesenchymal transition. Mol Cancer 13: 68, 2014.

56. Flockhart RJ, Webster DE, Qu K, Mascarenhas N, Kovalski J, Kretz $\mathrm{M}$ and Khavari PA: BRAFV600E remodels the melanocyte transcriptome and induces BANCR to regulate melanoma cell migration. Genome Res 22: 1006-1014, 2012.

57. Wajapeyee N, Serra RW, Zhu X, Mahalingam M and Green MR Oncogenic BRAF induces senescence and apoptosis through pathways mediated by the secreted protein IGFBP7. Cell 132: 363-374, 2008.

58. Goodall ML, Wang T, Martin KR, Kortus MG, Kauffman AL, Trent JM, Gately S and MacKeigan JP: Development of potent autophagy inhibitors that sensitize oncogenic BRAF V600E mutant melanoma tumor cells to vemurafenib. Autophagy 10 1120-1136, 2014

59. Sueda T, Sakai D, Kawamoto K, Konno M, Nishida N, Koseki J, Colvin H, Takahashi H, Haraguchi N, Nishimura J, et al: BRAF V600E inhibition stimulates AMP-activated protein kinasemediated autophagy in colorectal cancer cells. Sci Rep 6: 18949 2016.
60. Wang Y, Guo Q, Zhao Y, Chen J, Wang S, Hu J and Sun Y: BRAFactivated long non-coding RNA contributes to cell proliferation and activates autophagy in papillary thyroid carcinoma. Oncol Lett 8: 1947-1952, 2014

61. Viereck J, Kumarswamy R, Foinquinos A, Xiao K, Avramopoulos P, Kunz M, Dittrich M, Maetzig T, Zimmer K, Remke J, et al: Long noncoding RNA Chast promotes cardiac remodeling. Sci Transl Med 8: 326ra22, 2016.

62. Qi P, Zhou XY and Du X: Circulating long non-coding RNAs in cancer: Current status and future perspectives. Mol Cancer 15: 39, 2016.

63. Tiberio P, Callari M, Angeloni V, Daidone MG and Appierto V: Challenges in using circulating miRNAs as cancer biomarkers. BioMed Res Int 2015: 731479, 2015.

64. Dong L, Lin W, Qi P, Xu MD, Wu X, Ni S, Huang D, Weng WW, Tan C, Sheng W, et al: Circulating long RNAs in serum extracellular vesicles: Their characterization and potential application as biomarkers for diagnosis of colorectal cancer. Cancer Epidemiol Biomarkers Prev 25: 1158-1166, 2016.

65. Qu L, Ding J, Chen C, Wu ZJ, Liu B, Gao Y, Chen W, Liu F, Sun W, Li XF, et al: Exosome-transmitted lncARSR promotes sunitinib resistance in renal cancer by acting as a competing endogenous RNA. Cancer Cell 29: 653-668, 2016.

66. Rubinsztein DC, Codogno P and Levine B: Autophagy modulation as a potential therapeutic target for diverse diseases. Nat Rev Drug Discov 11: 709-730, 2012.

67. Cheng Y, Ren X, Hait WN and Yang JM: Therapeutic targeting of autophagy in disease: Biology and pharmacology. Pharmacol Rev 65: 1162-1197, 2013.

68. Høyer-Hansen M and Jäättelä M: Autophagy: An emerging target for cancer therapy. Autophagy 4: 574-580, 2008.

69. Wang Y, Hu Y, Sun C, Zhuo S, He Z, Wang H, Yan M, Liu J, Luan Y, Dai C, et al: Down-regulation of Risa improves insulin sensitivity by enhancing autophagy. FASEB J 30: 3133-3145, 2016. 